IP Periodica Polytechnica Civil Engineering

59(2), pp. 173 178, 2015

DOI: $10.3311 /$ PPci.7834

Creative Commons Attribution (1)

RESEARCH ARTICLE

\section{Analysis of Hyperbolic Transition Curve Geometry}

Zsolt Barna, Lajos Kisgyörgy

Received 24-11-2014, revised 04-12-2014, accepted 13-01-2015

\begin{abstract}
After the description of the basic geometry and relationships of the hyperbolic transition curve geometry we made further analysis. In this paper we analyzed the possible minimal length of the transition curve based on the dynamical characteristics. We determined the relationship between the dynamical parameters and the p parameter of the transition curve. For transition curves between tangential sections and curves we compared the minimal lengths of the hyperbolic geometry to the clothoide and cosine ones. The results show that according to the considered regulations the hyperbolic geometry gives shorter transition curve length. This has significant practical consequences as it makes the design more flexible.
\end{abstract}

\section{Keywords}

transition curve $\cdot$ third order motion characteristics $\cdot$ railway geometry

\section{Zsolt Barna}

Department of Highway and Railway Engineering, Budapest University of Technology and Economics, Múegyetem rkp. 3, H-1111 Budapest, Hungary e-mail: barna@uvt.bme.hu

\section{Lajos Kisgyörgy}

Department of Highway and Railway Engineering, Budapest University of Technology and Economics, Múegyetem rkp. 3, H-1111 Budapest, Hungary e-mail: kisgyorgy@uvt.bme.hu

\section{Introduction}

The transition curve geometries (clothoide, cosine, parabolic etc.) were developed several decades ago and no new transition geometry appeared since. Therefore there is no current research analyzing of the basic transition geometries. Instead researches focus along the well-known basic transition geometries on the examination of the vehicle-track complex system [8], evaluated the dynamic effects and suggested complex solutions for the curvature change [4].

Our research dealt with something new, a new transition geometry, the hyperbolic transition. Therefore we had to go back to the beginning, to the analysis of the basic characteristics of the new geometry. This way we were behind the current research activities in complexity, because we had to demonstrate the characteristics of the hyperbolic geometry - just like it had been done several decades ago for the other geometries. The detailed modeling of the track and vehicle relationship would be a further step after getting to know the basic characteristics of the geometry.

In our paper we compare the hyperbolic transition curve to two frequently applied transition geometries, to the clothoide and the cosine transition curves. Our research question was if the application of the S-shaped hyperbolic transition curve offered definite practical advantages over the application of the traditional geometries.

\section{Basic geometry of hyperbolic transition curve}

Based on the sine hyperbolic function [6] introduced the concept of the parametric hyperbolic transition curve and described the basic relationships. The curvature function of the hyperbolic geometry is:

$$
G(l)=\frac{1}{2 R} \frac{\sinh \left(p-\frac{2 p}{L} l\right)-\sinh (p)+\frac{2 p}{L} l \cosh (p)}{p \cosh (p)-\sinh (p)} .
$$

Fig. 1 shows the graphical representation of the curvature function. Figs. 2 and 3 display the third and fourth order motion characteristics of the geometry, which is characteristically similar to the first and second derivate of the curvature function. 
The calculations were done according to the basic theoretical approach described by Megyeri [7], using power series to describe the functions. The first two terms of the power series were used, because the numeric difference between the results of applying two or three terms are extremely slight, by several order under the accuracy of geodetic setting out.

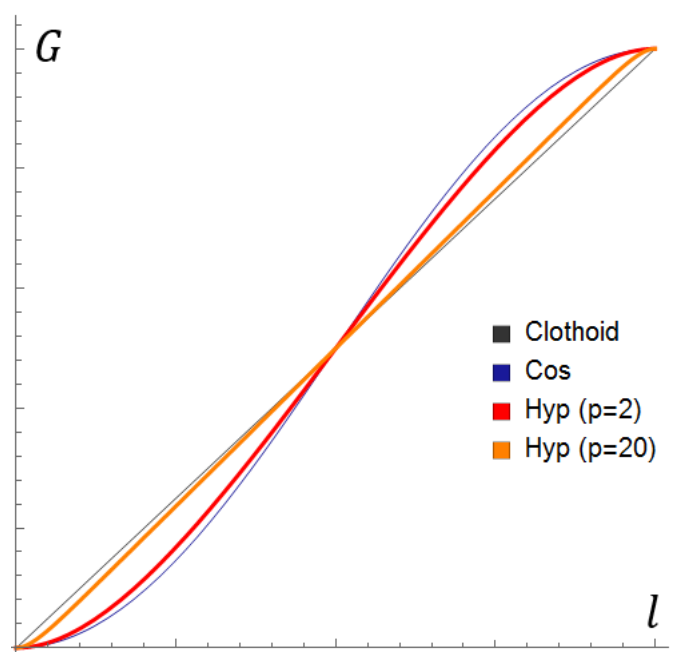

Fig. 1. The curvature function of the hyperbolic transition curve $(G)$

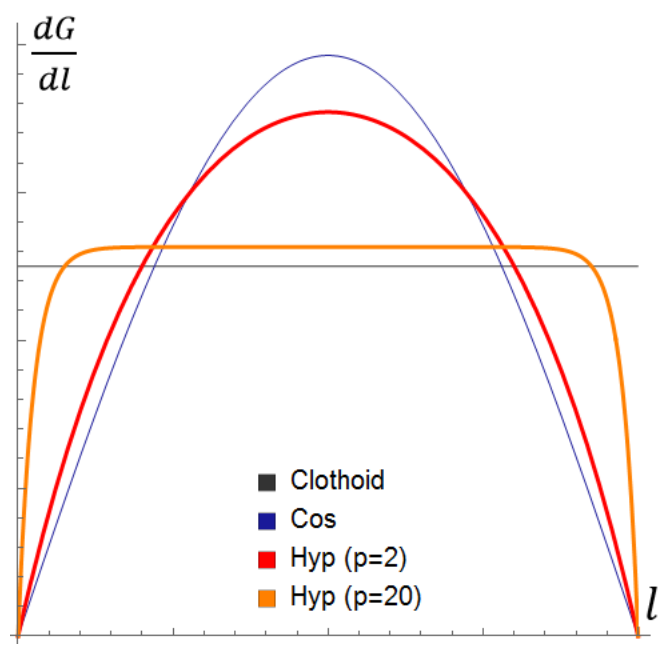

Fig. 2. Third order motion characteristic of the hyperbolic transition curve $(d G / d l)$.

\section{Determination of maximum change of acceleration in hyperbolic transition curve}

3.1 Third order motion characteristics of hyperbolic geometry

The minimum length of transition curves depends on the third order motion characteristics, as its value may not exceed the limiting value of vector $\bar{h}$. The vector $\bar{h}$ in general case can be described by the following function, as it is determined by Megyeri [7]:

$$
\bar{h}=\left(\frac{d a_{t}}{d t}-v^{3} G^{2}\right) \bar{t}+\left(3 v a_{t} G+v^{2} \frac{d G}{d t}\right) \bar{n}+v^{3} G T \bar{b}
$$

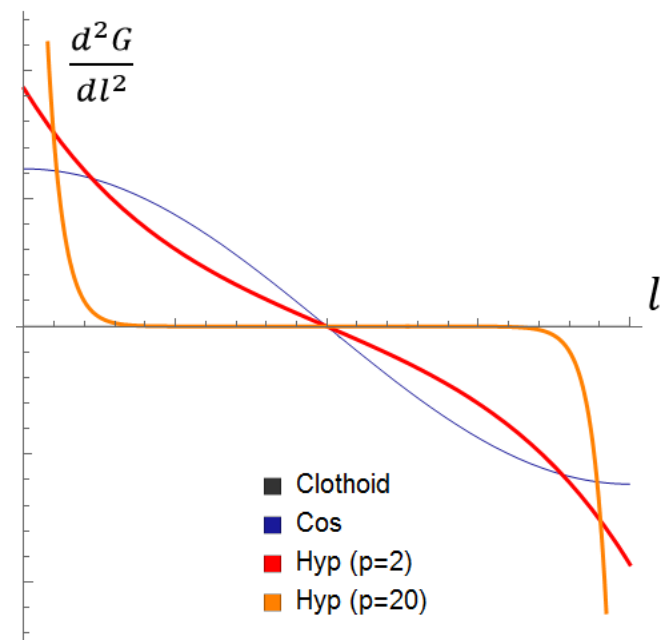

Fig. 3. Fourth order motion characteristic of the hyperbolic transition curve $\left(d^{2} G / d l^{2}\right)$

If acceleration is considered constant then the simplified function of vector $\bar{h}$ is:

$$
\bar{h}=-v^{3} G^{2} \bar{t}+\left(3 v a_{t} G+v^{3} \frac{d G}{d l}\right) \bar{n}+v^{3} G T \bar{b},
$$

where:

$\begin{array}{ll}v & \text { Speed }[\mathrm{m} / \mathrm{s}] \\ a_{t} & \text { Tangential acceleration }\left[\mathrm{m} / \mathrm{s}^{2}\right] \\ G & \text { Curvature of the track }[1 / \mathrm{m}] \\ T & \text { Torsion of the track }[1 / \mathrm{m}]\end{array}$

$\bar{t}, \bar{n}, \bar{b}$ The first three orthonormal unit vector of the Frenet frame

From the point of view of vector $\bar{h}$ the middle of the transition curve is the critical location (Fig. 2). Using parameterization by arc length this point is located at $l=L / 2$, where $L$ is the length of the transition curve. The curvature of hyperbolic geometry at location $l=L / 2$ is [6]:

$$
G=\frac{1}{2 R}
$$

The value of the first derivate of the curvature $(d G / d l)$ at $l=L / 2$ :

$$
\frac{d G}{d l}(l=L / 2)=\frac{p}{R L} \frac{\cosh (p)-1}{p \cosh (p)-\sinh (p)} .
$$

\subsection{Torsion of hyperbolic geometry}

The torsion of a curve measures how sharply it is twisting out of the plane of curvature. We determined the torsion of the hyperbolic geometry based on the parametric equation of the space curve [7]:

$$
\bar{r}(l)=x(l) \bar{i}+y(l) \bar{j}+z(l) \bar{k} .
$$

where

$\bar{r}(l) \quad$ The position vector parameterized by arc length $x(l), y(l)$ Horizontal orthogonal coordinates 
Vertical orthogonal coordinate

Torsion can be computed from the following formula:

$$
T(l)=\frac{\frac{d r}{d s} \frac{d^{2} r}{d s^{2}} \frac{d^{3} r}{d s^{3}}}{G^{2}}=\frac{\left|\begin{array}{ccc}
x^{\prime} & y^{\prime} & z^{\prime} \\
x^{\prime \prime} & y^{\prime \prime} & z^{\prime \prime} \\
x^{\prime \prime \prime} & y^{\prime \prime \prime} & z^{\prime \prime \prime}
\end{array}\right|}{x^{\prime \prime 2}+y^{\prime \prime 2}+z^{\prime \prime 2}} .
$$

At the calculation of $x(l)$, and $y(l)$ coordinates we applied to approximations:

- The horizontal coordinates were approximated by the first terms of the function series of cosine and sine functions (this simplification was used only at the calculation of torsion):

$$
\begin{gathered}
x(l)=\int_{0}^{l} \cos \left(\tau_{l}\right) d l=\int_{0}^{l} d l, \\
y(l)=\int_{0}^{l} \sin \left(\tau_{l}\right) d l \approx \int_{0}^{l} \tau_{l} d l \approx l .
\end{gathered}
$$

- The curvature of the space curve was approximated by the curvature of $(x, y)$ plane because the difference is negligible from practical aspects.

Superelevation development, which is located inside the transition curves, influence the value of vector $\bar{h}$ through torsion. In this paper we used the cosine geometry for superelevation development for the hyperbolic geometry as well:

$$
z(l)=\frac{m}{2}\left(1-\cos \left(\frac{\pi}{L} l\right)\right) .
$$

In the equation $m$ denotes the value of superelevation at the end of the transition curve. It was determined by the formula applied in Hungary:

$$
m=11.8 \frac{V^{2}}{R}-100
$$

where

$\begin{array}{ll}m & \text { Superelvation at the end of the transition curve }[\mathrm{mm}] \\ V & \text { Speed }[\mathrm{km} / \mathrm{h}] \\ R & \text { Radius of the curve }[\mathrm{m}]\end{array}$

At the $l=L / 2$ critical point the value of torsion will trivially be identical with cosine transition curve - cosine superelevation development configuration:

$$
T=-\frac{m R \pi^{3}}{2 L^{3}}
$$

\subsection{Lateral acceleration change in hyperbolic geometry}

The absolute value of the third order motion characteristic was determined based on (Eq. (3)):

$$
|\bar{h}|=\sqrt{-v^{6} G^{4}+\left(3 v a_{t} G+v^{3} \frac{d G}{d l}\right)^{2}+v^{6} G^{2} T^{2}}
$$

Substituting (Eqs. 44, 5, (12) into (Eq. 13) and converting speed to $\mathrm{km} / \mathrm{h}$ dimension $(v[\mathrm{~m} / \mathrm{s}]=V[\mathrm{~km} / \mathrm{h}] / 3,6)$ the lateral acceleration change in the critical point is:

$$
\left|\bar{h}\left(\frac{L}{2}\right)\right|=\sqrt{\frac{m^{2} \pi^{6} V^{6}}{16 \cdot 3,6^{6} L^{6}}+\frac{V^{6}}{16 \cdot 3,6^{6} R^{4}}+}
$$

$$
+\frac{\left(2 p V^{3}(1-\cosh (p))+3 a \pi^{2} L V(\sinh (p)-p \sinh (p))\right)^{2}}{4 \pi^{6} L^{2} R^{2}(\sinh (p)-p \sinh (p))^{2}}
$$

\section{Determination of the minimal transition curve length}

\subsection{Basic approach}

The practical goal in railroad design is to apply the shortest possible transition curves which fulfills the boundary conditions. The minimal transition curve length for speed-curve radius relations can be determined by (Eq. (14)). Thus theoretically the relationship between the $L$ length of the hyperbolic transition curve and the $p$ parameter is defined. But there are further practical aspects as well:

- The coordinates were expressed as power series, so it makes sense to check how the extreme values of parameter $\mathrm{p}$ effects the accuracy of the coordinates.

- The vehicle entering into the transition geometry begins to feel the transition when its pins start to deviate away the tangent. We analyzed the parameter values from the point of view of smooth transition, where the center of mass of the vehicle deviates only in the transition curve. Here the accuracy of geodetic setting out and the vehicle characteristics were considered. The geometrical tolerancies of the track right after the construction are normally $1-2 \mathrm{~mm}$, therefore - similarly to the spline-method [1] - we do not suppose that the real railway track fits absolutely the calculated coordinates. Therefore we only considered the deviation effect of the transition curve to exist if the value of the ordinate exceeded a chosen minimal value $\left(y_{\min }\right)$, so the calculations were made for $y_{\min }=0.5 \mathrm{~mm}$ and $y_{\min }=1 \mathrm{~mm}$ geodetic accuracy.

As it is demonstrated by Bocz [2], the Hungarian legislation applies a multi-level approach to track alignment design:

- The Hungarian National Railway Regulation [10] is harmonized to the valid draft Technical Specification of Interoperability (TSI). This regulation defines the normal limit of the lateral acceleration change as $h=0.4 \mathrm{~m} / \mathrm{s}^{3}$.

- The Track Alignment Design Regulation of National Public Railways [9] contains more detailed regulation of alignment design. It defines the exact length of transition curves, depending on alignment speed, and sets limits for the maximum value of the lateral acceleration changes as well. This values are $h_{1}=0.4 \mathrm{~m} / \mathrm{s}^{3}$, for cosine and $h_{2}=0.3 \mathrm{~m} / \mathrm{s}^{3}$ for clothoide transition curves. 
Common element of the two regulations is that both of them contain the linear (clothoid) and S-shaped (cosine) transition geometry. However the OVSZ gives only the maximal values of allowable lateral acceleration, of its change and of superelevation, and says nothing about the minimal radii of curves. The OKVPSZ contains several definitions for the radii of curves, from which we used two in our analysis:

$R_{1} \quad-$ suggested minimal radii of curve in the function of alignment speed,

$R_{2} \quad-$ minimum allowed radius of curve in function of speed in case of curve correction of existing lines.

In our calculation we used the following values for lateral acceleration and its change according to the Hungarian regulation:

- In Hungary there are two limit values for the change of lateral acceleration as it was mentioned above. We made the calculation for both of them:

- $h_{1}=0.4 \mathrm{~m} / \mathrm{s}^{3}$ (general case);

- $h_{2}=0.3 \mathrm{~m} / \mathrm{s}^{3}$ (in case of clothoide geometry).

- For lateral acceleration $a_{t}=0.35 \mathrm{~m} / \mathrm{s}^{2}$ value was used.

\subsection{Analysis of parameter $p$}

The shape of hyperbolic transition curves depends on the parameter $p$. By changing this parameter the shape can be modified continuously between the clothoide and the cosine transition curve. By increasing the value of $p$ the hyperbolic geometry approaches the clothoide one and its characteristics more and more. By decreasing the value of $p$ the hyperbolic geometry gets very close to the cosine transition curve.

At the analysis of smaller and smaller values of $p$ we experienced that below $p \approx 0.1 . .0 .2$ values - depending on the values of $R$ and $L$ - the values of $y$ ordinate are scattered in a wide lane around the theoretical curve of the transition geometry (Fig. 4). This makes necessary to choose a minimal value for parameter $p$ for practical purposes, and we applied $p_{\min }=1$ minimal value. This question can require possible further research, but from the point of view of practical application of hyperbolic geometry is irrelevant.

We analyzed the effect of high parameter values to the transition curve geometry. The higher the parameter the shorter the transition curve and the lower the $y$ ordinate of the end point of the transition curve $(Y)$ is. However the higher the $p$ parameter is the less extent of $y$ ordinate will decrease. The limiting value of the $y$ ordinate of the end point at $p \rightarrow \infty$ :

$$
\lim _{p \rightarrow \infty} Y_{\text {hyp }}=\frac{56 L^{2} R^{2}-L^{4}}{336 L^{3}}
$$

The results showed that above $p=100$ the value of $Y$ end point ordinate could be decreased by only $\sim 1 \mathrm{~cm}$ at most, which is negligible in practical applications. This way the maximal value of parameter $p$ was set as $p_{\max }=100$.
The minimal length of the hyperbolic transition can be determined based on (Eq.(14), but no explicit formula can be developed to describe the limiting value of $L$ at $p \rightarrow \infty$. We made calculations for speed-curve radius $(V-R)$ pairings taken from practical applications. The result showed that above $p_{\max }=100$ only $\sim 1 \mathrm{~m}$ decrease of the length could be achieved. As it is less than $1 \%$ of the shortest transition curve lengths applied in practice the $p_{\max }=100$ maximal value appropiate from this aspect as well.

\subsection{Perceptibility of the hyperbolic geometry}

In this stage of research we analyzed the basic geometry of hyperbolic transition curves so we considered the vehicle as a moving point on a path. Based on this we assumed the vehicle perceived the transition curve when its motion sensing point entered into the geometry. The motion sensing point of the vehicle is the middle point between king pins, and the general distance between pins is $d=17 \mathrm{~m}$. Our goal was to apply only those transitions geometries where the motion sensing point of the vehicle does not deviate from the tangent outside the transition curve. This meant transition curves whose $y$ ordinates at $d / 2$ length of the transition curve did not exceed the geodetic setting out accuracy ( 0.5 and $1.0 \mathrm{~mm}$, respectively).

In our calculations we used both the $y_{\min }=0.5 \mathrm{~mm}$ constructional accuracy and $y_{\min }=1.0 \mathrm{~mm}$ maintenance accuracy. In case of hyperbolic geometry the higher the parameter is the faster the value of $y$ ordinates increase, so we defined the parameter where the $y$ ordinate at $d / 2$ length equals to $y_{\min }$ as maximal from the aspect of vehicle motion. If the $y$ ordinate of hyperbolic transition geometry with $p=100$ parameter did not exceed $y_{\min }$, then this $p=100$ parameter was set as maximal, according to the earlier reasoning.

\subsection{Results}

In our calculations we determined the minimal length of the hyperbolic transition curve inside the $120-160 \mathrm{~km} / \mathrm{h}$ speed interval, using $R_{1}$ and $R_{2}$ minimal curve radii and $h_{1}$ and $h_{2}$ maximal changing of acceleration values according to the Hungarian regulation. The results are shown in Table 1 for $1.0 \mathrm{~mm}$ geodetic accuracy and in Table 2 for $0.5 \mathrm{~mm}$ geodetic accuracy.

The minimal length of the clothoide and the cosine transitions geometries [5,7] were calculated also for these values.

\section{Conclusions and future development possibilities}

The results show that the hyperbolic transition geometry requires shorter transition curves than the other ones. In case of $h_{1}=0,4 \mathrm{~m} / \mathrm{s}^{3}$ maximal lateral acceleration change according to OVSZ the differences are significant, so the application of the hyperbolic transition geometry has definite practical advantages, especially at severe geometrical restrictions.

The OKVPSZ gives lower allowances for the lateral acceleration change, here $h_{2}=0,3 \mathrm{~m} / \mathrm{s}^{3}$ is applied. This restriction regards to the clothoide geometry because of the incontinuity of 


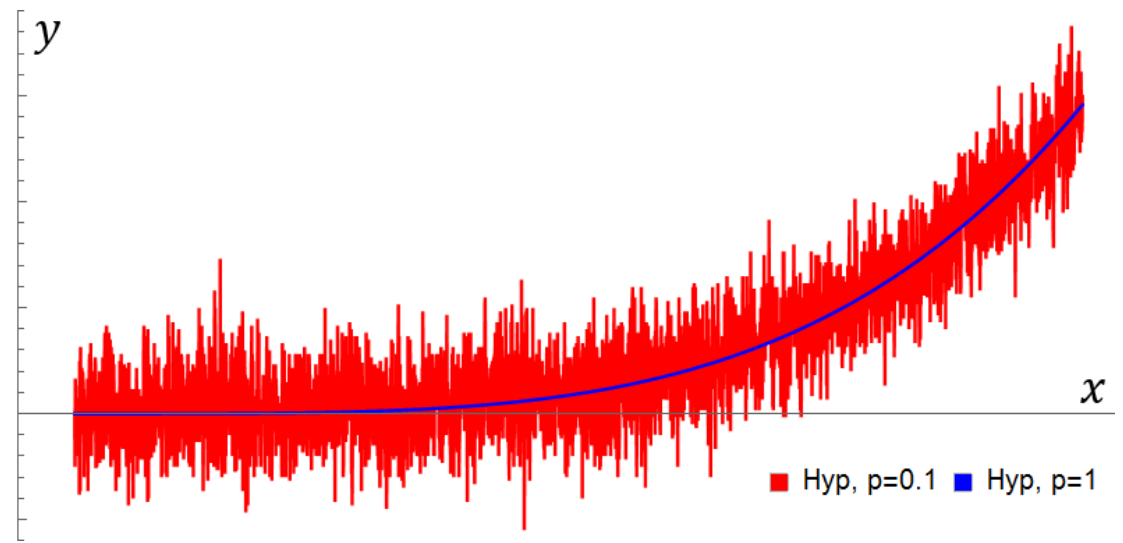

Fig. 4. Disintegration of transition geometry in case of low values of $p$

Tab. 1. Comparison of minimal lengths of transition curves in case of $1.0 \mathrm{~mm}$ geodetic accuracy.

\begin{tabular}{|c|c|c|c|c|c|c|c|}
\hline \multirow{2}{*}{$V_{t}[\mathbf{k m} / \mathbf{h}]$} & \multirow{2}{*}{$R_{1}[\mathrm{~m}]$} & \multicolumn{2}{|c|}{$h_{1}=0.4 \mathrm{~m} / \mathrm{s}^{3}$} & \multicolumn{2}{|c|}{$h_{2}=0.3 \mathrm{~m} / \mathrm{s}^{3}$} & \multirow{2}{*}{$L_{\cos }[\mathrm{m}]$} & \multirow{2}{*}{$L_{\text {clothoid }}[\mathrm{m}]$} \\
\hline & & $\mathbf{p}[-]$ & $\mathrm{L}_{\text {hyp }}[\mathrm{m}]$ & $\mathbf{p}[-]$ & $\mathrm{L}_{\text {hyp }}[\mathrm{m}]$ & & \\
\hline 120 & 1400 & 100 & 69.05 & 100 & 93.00 & 107.26 & 96.43 \\
\hline 130 & 1600 & 100 & 76.70 & 100 & 103.23 & 119.12 & 106.25 \\
\hline 140 & 1800 & 100 & 85.05 & 100 & 114.41 & 132.08 & 118.33 \\
\hline 150 & 2000 & 100 & 94.06 & 100 & 126.47 & 146.05 & 132.00 \\
\hline 160 & 2200 & 100 & 103.70 & 100 & 139.38 & 161.01 & 145.45 \\
\hline \multirow{2}{*}{$V_{t}[\mathbf{k m} / \mathbf{h}]$} & \multirow{2}{*}{$R_{2}[\mathrm{~m}]$} & \multicolumn{2}{|c|}{$h_{1}=0.4 \mathrm{~m} / \mathrm{s}^{3}$} & \multicolumn{2}{|c|}{$h_{2}=0.3 \mathrm{~m} / \mathrm{s}^{3}$} & $I \quad[\mathrm{ml}$ & $I$ \\
\hline & & $\mathbf{p}[-]$ & $\mathrm{L}_{\text {hyp }}[\mathrm{m}]$ & $\mathbf{p}[-]$ & $\mathrm{L}_{\text {hyp }}[\mathrm{m}]$ & $\mathrm{L}_{\cos }[\mathbf{m}]$ & $L_{\text {clothoid }}$ [II] \\
\hline 120 & 950 & 100 & 103.39 & 100 & 140.01 & 160.57 & 142.11 \\
\hline 130 & 1100 & 100 & 113.17 & 100 & 153.08 & 175.75 & 154.55 \\
\hline 140 & 1300 & 100 & 119.12 & 100 & 160.90 & 184.99 & 163.85 \\
\hline 150 & 1500 & 100 & 126.61 & 100 & 170.82 & 196.61 & 176.00 \\
\hline 160 & 1700 & 100 & 135.28 & 100 & 182.37 & 210.07 & 188.24 \\
\hline
\end{tabular}

Tab. 2. Comparison of minimal lengths of transition curves in case of $0.5 \mathrm{~mm}$ geodetic accuracy.

\begin{tabular}{|c|c|c|c|c|c|c|c|}
\hline \multirow{2}{*}{$V_{t}[\mathbf{k m} / \mathbf{h}]$} & \multirow{2}{*}{$R_{1}[\mathrm{~m}]$} & \multicolumn{2}{|c|}{$h_{1}=0.4 \mathrm{~m} / \mathrm{s}^{3}$} & \multicolumn{2}{|c|}{$h_{2}=0.3 \mathrm{~m} / \mathrm{s}^{3}$} & \multirow{2}{*}{$L_{c o s}[\mathrm{~m}]$} & \multirow{2}{*}{$L_{\text {clothoid }}[\mathrm{m}]$} \\
\hline & & $\mathbf{p}[-]$ & $L_{\text {hyp }}[\mathrm{m}]$ & $\mathbf{p}[-]$ & $\mathrm{L}_{\text {hyp }}[\mathrm{m}]$ & & \\
\hline 120 & 1400 & 14 & 73,58 & 32,5 & 94,98 & 107,26 & 96,43 \\
\hline 130 & 1600 & 23,5 & 79,28 & 78 & 103,52 & 119,12 & 106,25 \\
\hline 140 & 1800 & 47,5 & 86,00 & 100 & 114,41 & 132,08 & 118,33 \\
\hline 150 & 2000 & 100 & 94,06 & 100 & 126,47 & 146,05 & 132,00 \\
\hline 160 & 2200 & 100 & 103,70 & 100 & 139,38 & 161,01 & 145,45 \\
\hline \multirow{2}{*}{$V_{t}[\mathbf{k m} / \mathbf{h}]$} & \multirow{2}{*}{$R_{2}[\mathrm{~m}]$} & \multicolumn{2}{|c|}{$h_{1}=0.4 \mathrm{~m} / \mathrm{s}^{3}$} & \multicolumn{2}{|c|}{$h_{2}=0.3 \mathrm{~m} / \mathrm{s}^{3}$} & & \\
\hline & & $\mathbf{p}[-]$ & $L_{\text {hyp }}[\mathrm{m}]$ & $\mathbf{p}[-]$ & L $_{\text {hyp }}[\mathrm{m}]$ & $L_{\cos }[\mathbf{m}]$ & $L_{\text {clothoid }}[\mathbf{m}]$ \\
\hline 120 & 950 & 20,5 & 107,56 & 51 & 141,37 & 160,57 & 142,11 \\
\hline 130 & 1100 & 35,5 & 115,26 & 100 & 153,08 & 175,75 & 154,55 \\
\hline 140 & 1300 & 69 & 119,65 & 100 & 160,90 & 184,99 & 163,85 \\
\hline 150 & 1500 & 100 & 126,61 & 100 & 170,82 & 196,61 & 176,00 \\
\hline 160 & 1700 & 100 & 135,28 & 100 & 182,37 & 210,07 & 188,24 \\
\hline
\end{tabular}


the curvature function of the geometry. If we apply this restriction to the hyperbolic geometry as well then it will be shorter than the clothoide but only marginally. However the curvature function of the hyperbolic transition geometry is smooth and continuous, so the application of $h_{2}=0,3 \mathrm{~m} / \mathrm{s}^{3}$ value is not reasonable. However the clarification of this question requires further research.

The hyperbolic transition geometry offers a flexible and effective possibility, which opens numerous new research questions. We plan to make further analysis to get more information about this new geometry. Some of them connects to actual analysis of other transition curve geometries, such as:

- Hyperbolic transition geometry as superelevation development geometry [2]. In this research the vehicle will be considered as a space body.

- The examination of the shape of the geometry based on the geodetic setting out coordinates by the novel spline theory.

- The examination of the characteristics of hyperbolic transition curve in the legislation frame [3], especially from the point of view of application above $160 \mathrm{~km} / \mathrm{h}$ design speed.

- From practical considerations it could be very important to analyze the application of the hyperbolic transition geometry in track reconstructions of speed improvement. It is expected that this new geometry makes less earthwork construction needed.

\section{References}

1 Andor K, Polgár R, Localization of bearing errors using spline method, Periodica Polytechnica Civil Engineering, 58(4), (2014), DOI 10.3311/PPci.7528

2 Bocz P, Examination of transition curves by detailed kinematical methods, Magyar Építóipar, 1, (2014), 8-15.

3 Fischer S, Comparison of railway track transition curves, Pollack Periodica, 4(3), (2009), 99-110.

4 Hasslinger HL, Gleis mit Übergangsbogen und kräfteminimaler Überhöhungsrampe. Österreichische Patentschrift AT 412975 B.

5 Kerkápoly E, Megyeri J, Tables for railway track aligning (in Hungarian), Múszaki Könyvkiadó; Budapest, 1980, ISBN 9631027589.

6 Kisgyörgy L, Barna Z, Hyperbolic transition curve, Periodica Polytechnica Civil Engineering, 58(1), (2014), 63-69, DOI 10.3311/PPci.7433

7 Megyeri J, Railway movement geometry (in Hungarian), Múszaki Könyvkiadó; Budapest, 1986, ISBN 9631059782.

8 Zboiński K, Woźnica P, Optimisation of the Railway Transition Curves' Shape with Use of Vehicle-track Dynamical Model, The Archives of Transport, 22(3), (2010), 387-407, DOI 10.2478/v10174-010-0024-z

9 Track alignment design regulation of national public railways (Hungarian: Országos Közforgalmú Vasutak Pályatervezési Szabályzata, OKVPSZ), KÖZDOK, 1983.

10 103/2003. (XII. 27.) Decree of Ministry of Transport on the interoperability of the conventional rail system. Annex 4. National Railway Regulation (Hungarian: Országos Vasúti Szabályzat, OVSZ), 2003. 